\title{
Study of the binding mechanisms between palytoxin and its aptamer by docking and molecular simulation
}

\author{
Bo Hu${ }^{1,2 \pi}$, Rong Zhou ${ }^{1,2 \pi}$, Zhengang $\mathrm{Li}^{1,2 \pi}$, Jiaxiang Qin ${ }^{3}$, Shengqun Ouyang ${ }^{1}$, Zhen $\mathrm{Li}^{1}$, \\ Wei Hu ${ }^{3}$,Lianghua Wang ${ }^{1 *}$, Binghua Jiao ${ }^{1,2 *}$
}

${ }^{1}$ Department of Biochemistry and Molecular Biology, College of Basic Medical, Second Military Medical University, Shanghai 200433, China.

${ }^{2}$ Marine Biological Institute, College of Marine Military Medicine, Second Military Medical University, Shanghai 200433, China.

${ }^{3}$ Chengdu FenDi Technology Co., Ltd, Chengdu 610041, China.

${ }^{*}$ Corresponding author

E-mail: Ihwang@smmu.edu.cn (LW); bhjiao@smmu.edu.cn(BJ)

IThese authors contributed equally to this work.

\begin{abstract}
This paper provides a feasible model for aptamer and its target in molecular structure analysis and interaction mechanism. In this study, modeling and dynamic simulation of ssDNA aptamer ( $P$ 18S2) and target (Palytoxin,PTX) were performed separately. Then, the combination mechanism of DNA and PTX were predicted, and docking results showed that PTX can combine steadily in the groove at the top of DNA model trough strong hydrogen-bonds and electrostatic interaction. Therefore, we have further truncated and optimized to P-18S2 by simulating, at the same time, we also confirmed the reliability of simulative results by experimenting. Combining with the experimental and computational results, we provide a more reasonable interpretation for the high affinity and specific binding of P-18S2 and PTX and offer the basis for aptamer development in molecular diagnostics and therapeutic application.
\end{abstract}

\section{Author summary}

In order to further study the complex structure and interaction of P-18S2 and PTX, a series of molecular modeling program were designed, including modeling, traditional dynamics simulation and molecular docking. Modeling results reveal that the structure of P-18S2 is a DNA G-quadruplex. Meanwhile, the 3D structure of PTX with lowest total energy after equilibrium was selected to use for the subsequent simulations. Then, based on the DNA with G-quadruplex structure, the combination model of DNA and PTX were predicted, and docking results showed that PTX can combine steadily in the groove at the top of DNA model trough strong hydrogen-bonds and electrostatic interaction. Futhermore, we compared the affinity of 6 optimized aptamers by computer simulating to primary P-18S2 bind to PTX respectively, the results showed no significant difference. Therefore, we have further truncated and optimized to P-18S2. In addition, this paper further refined research method based on our previous study ${ }^{[17]}$, the instability of the target structure was considered and optimized, and the biological experiments were used to confirm the veracity of the simulative results. Combining with the experimental and computational methods, we obtained a reasonable interpretation for the high affinity and specific binding of P-18S2 and PTX. In summary, 
we established a feasible model for aptamer and its target in molecular structure analysis and interaction mechanism, and offer the basis for the study of aptamer development in molecular diagnostics and therapeutic application.

\section{Introduction}

Palytoxin (PTX) is one of the most powerful non-protein natural toxin, which was first separated from soft corals in $1971^{[1]}$. The LD50 of PTX after intraperitoneal injection is $25 \mathrm{ng} / \mathrm{kg}$ and $50 \mathrm{ng} / \mathrm{kg}$ in rabbits and mice respectively[2]. Furthermore, PTX can cause dizziness, weakness, muscle pain, breathing difficulties, heart failure, and even death in humans by ingesting seafood contaminated with PTX or direct contact with aerosolized water during dinoflagellate blooms ${ }^{[3-5]}$. Fortunately, the problem of affecting human health and global shellfish industry development due to the contamination of shellfish by PTX has attracted great attention. Meanwhile, many detection methods for PTX have been developed such as mouse bioassay[6], liquid chromatography coupled to a fluorometer, ultraviolet-visible spectrophotometer, or mass spectrometer ${ }^{[7-9]}$. However, there are still many challenges such as the ethical issues and the expensive instruments. We have also been working on PTX research and successfully obtained a DNA aptamer named P18S2(GGTGGGTCGGACGGGGGTGG), that can bind to PTX with high affinity and specificity, which could serve as a molecular recognition element in diagnosis and biological activity inhibition assays for PTX[10].

Aptamers are functional single-stranded DNA or RNA oligo nucleotides, which are selected from a random oligonucleotide libraries through systematic evolution of ligands by the exponential enrichment (SELEX) technique ${ }^{[11]}$. These aptamers have been isolated and adopted as diagnostic or therapeutic tools, can bind to various targets with high affinity and specificity by folding into steady and particular three-dimensional structures through intermolecular interactions such as the stacking of aromatic rings, electrostatic, van der Waals interactions, hydrogen bonds and induced fit mechanisms ${ }^{[12,}{ }^{13]}$. The development of aptamers offer a new opportunity to overcome the challenges of traditional methods for detecting toxins and address the risks of seafood and water contaminated with toxins. There are numerous aptamers have been developed into a novel detection method for toxins[14-16]. At the same time, the interaction mechanism of aptamers with targets still needs further exploration for the therapeutic application of aptamers. In our previous work, we obtained the aptamer(GO18-T-d) $)^{[15]}$ of GTX1/4 and analysed the binding mechanism of GTX1/4 and G018-T-d by a series of molecular modeling programs ${ }^{[17]}$. In addition, we also obtained the aptamer P18-S2 that can bind to PTX with a high Kd of $0.93 \mathrm{nM}^{[10]}$ by SELEX and Biolayer interferometry (BLI) which is a real-time optical analytical technique for measuring interactions between biomolecule ${ }^{[18]}$. In this study, we also designed a series of molecular modeling programs in order to research and further interpret the binding mechanism between P-18S2 and PTX for the therapeutic application of aptamers.

\section{Results}

\section{Analysis of docking}

After modeling and optimization, the 3D structure of DNA P-18S2 with G-quadruplex structure was used as receptor in the docking. The combination of receptor with ligands was evaluated by the Etotal, Eshape and Eforce in the docking results. The electrostatic energy, Eforce, and the steric complementarity score, Eshape, were combined to give a total energy (Etotal) for the complex (in $\mathrm{kJ} / \mathrm{mol}$ units) ${ }^{[19]}$. In our Hex interaction simulation, the lower value of Etotal would result in more stable combination between DNA and PTX. As is shown in the S1 table, the total calculated 
interaction energy was listed, which showed the best energy ranked results. The observed best interaction energy and steric complementarity score of binding of DNA P-18S2 with PTX were calculated to be $-509.9 \mathrm{kcal} \mathrm{mol}-1$ and $-453.4 \mathrm{kcal}$ mol-1 respectively, indicated that the combination of P-18S2 and PTX was extremely stable, and well match in shape (Fig 1). Furthermore, electrostatic energy from P-18S2 and PTX was much lower, led to the higher value of interaction energy and more stable combination between P-18S2 and PTX.

In order to understand the interaction between DNA and ligands, some deeply analysis were performed to the top-ranking docked conformation of complex. As is shown in the Fig 1, the PTX inserted in docking site at the top of DNA P-18S2, made the combination stable. Quantitative analysis of $\mathrm{H}$-bond distance was performed, shown in Fig 2. There are six $\mathrm{H}$-bonds generate in complex from P-18S2 and PTX. It was worth to note that the combination between P-13S2 and PTX showed more H-bonds interaction from Bases of G1、G5、G9 and G13 and ligand, increased the stability of complex to a great extent (Fig 2). Therefore, P-18S2 showed a great activity to the obstruction of the binding between Sodium channel protein and PTX.
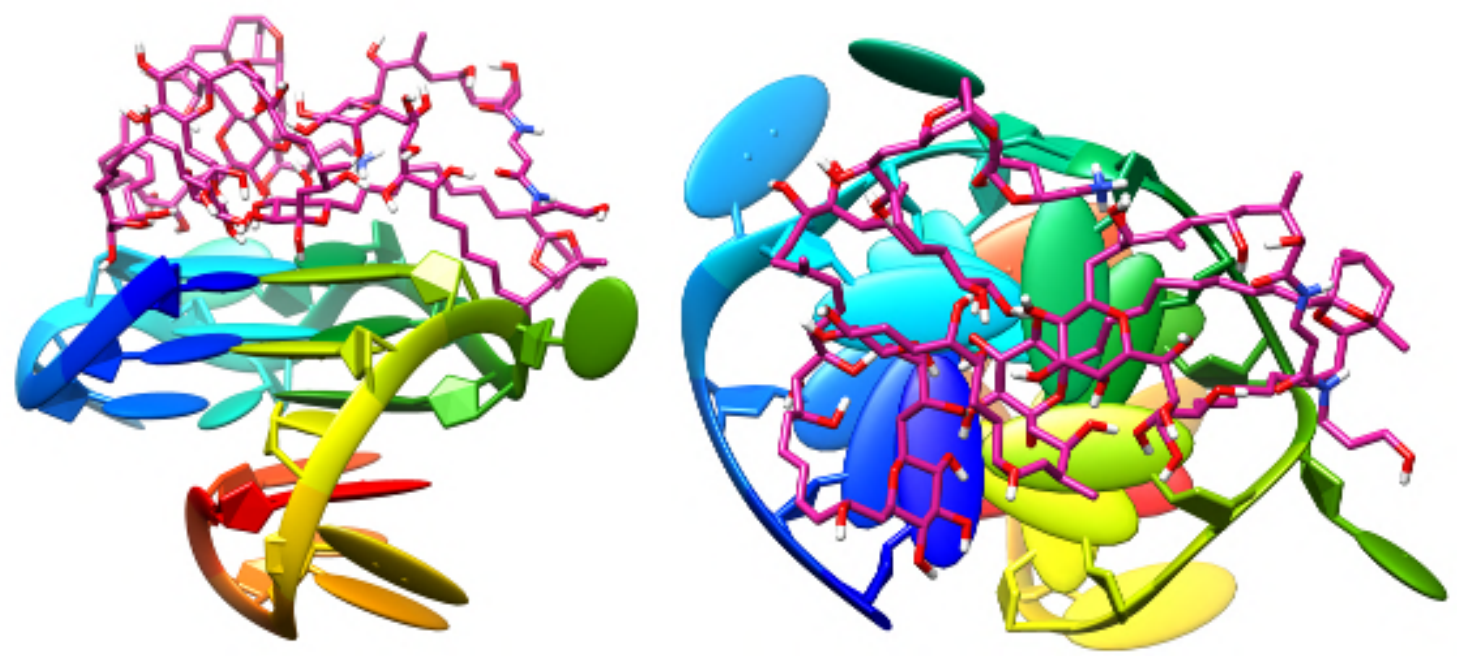

Figure 1 Three dimensional side view (A) and top view (B) of the top-ranking docked conformations between P-18S2 and PTX 


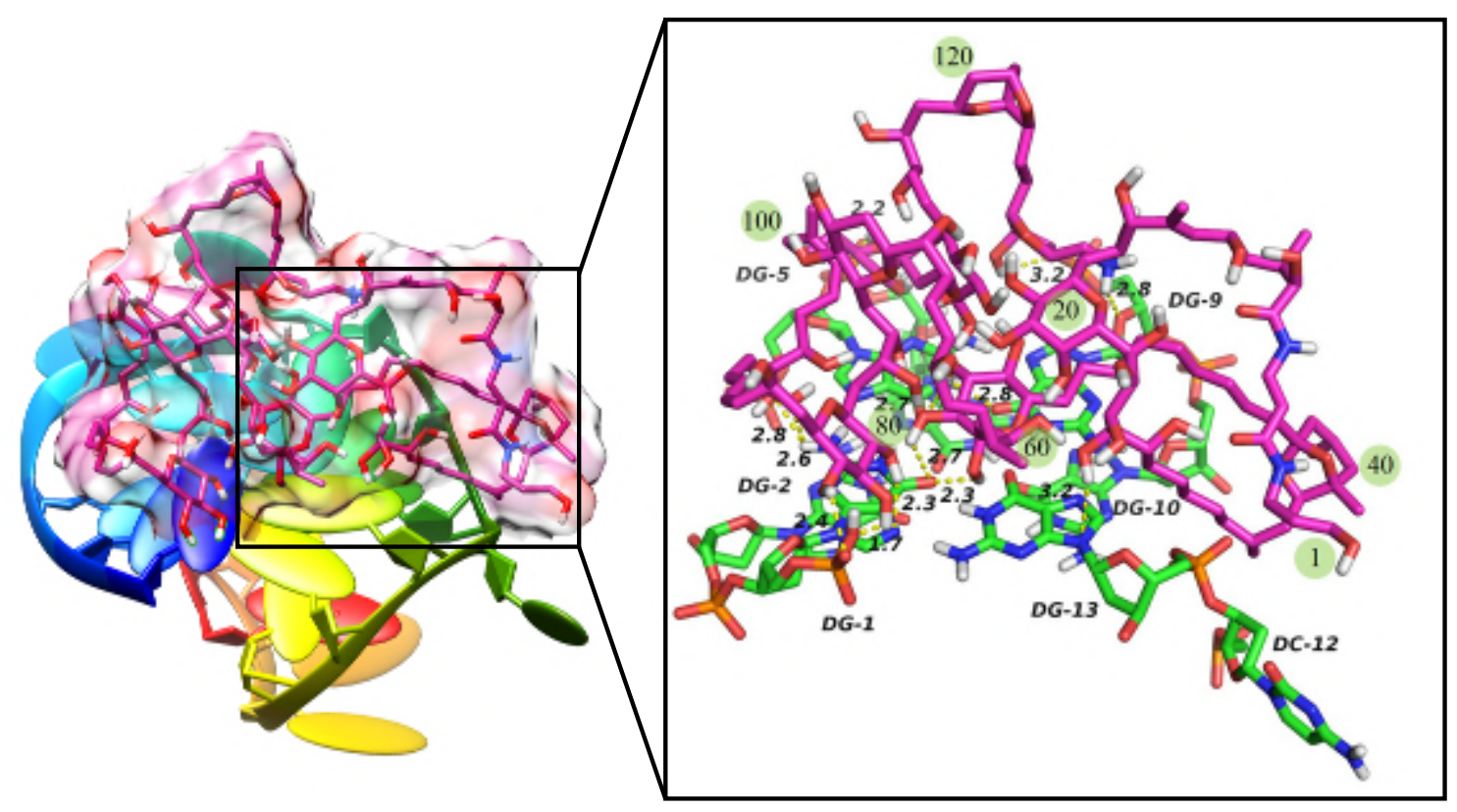

Figure 2 Three dimensional view of the interaction between P-18S2 and PTX

\section{Analysis of molecular dynamics simulations}

A common way to analyze the structural stability of a biomacromolecular in MD simulation is to monitor the root-mean-square deviation (RMSD) from the initial structure along the simulation. The RMSD of the complexes in this study are shown in Fig 3. It is clear from that plots that after $2 \mathrm{~ns}$ the complex of P-18S2-PTX reached an equilibrium state with fluctuation of $2 \AA$. Furthermore, in $30 \mathrm{~ns}$ of simulation the structures were inconsiderably distinct from the initial structures that were employed as the starting point of the simulations, shown in Figs 4 and 5, indicating that the complexes were stable. The conformation of complex with different time from 0ns, 10ns, 20ns and 30ns were similar, without isolation from each other. The mass distance of G-quadruplex structures and PTX had also demonstrated it, shown in Fig 6. It is clear that there is no obvious fluctuation in the plots of mass distance between DNA and PTX versus simulation time. In addition, the binding energy (Fig 7) of complexes P-18S2-PTX kept equilibrated, indicated that there is no separation between the DNA and PTX, kept combined steadily. If there is dissociation, the energy will fluctuate severely. The absence of fluctuation showed the strong non-bond interaction between DNA and PTX, which made the complexes stable. Consequently, the high affinity between PTX and DNA P$18 S 2$ blocked the binding between Sodium channel protein and PTX. 


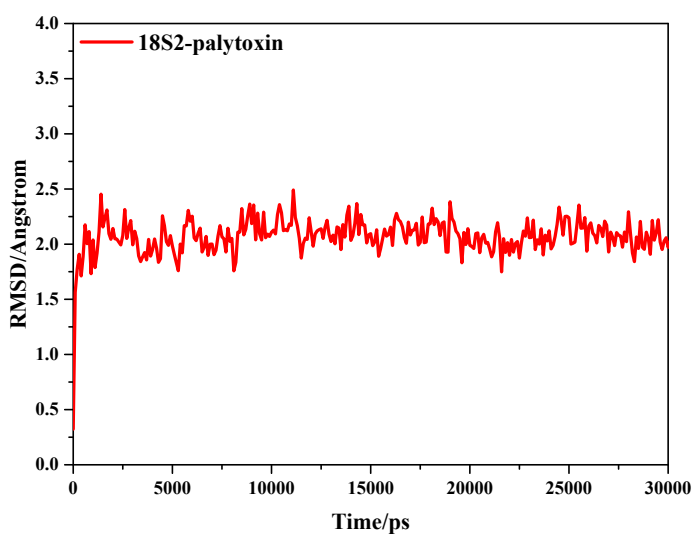

Figure 3 RMSD of CA atoms versus simulation time

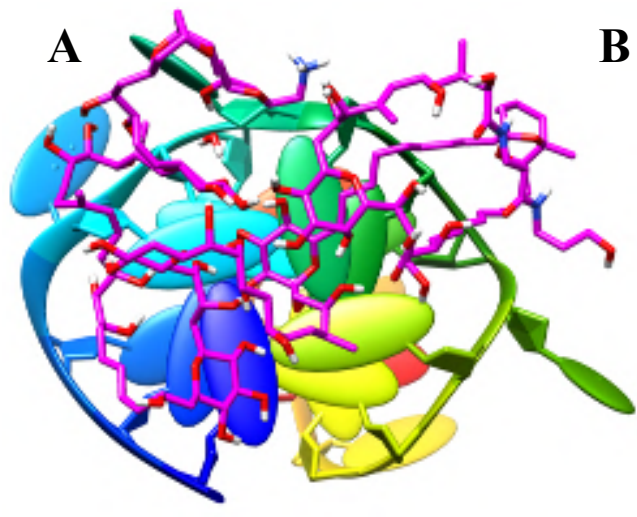

B

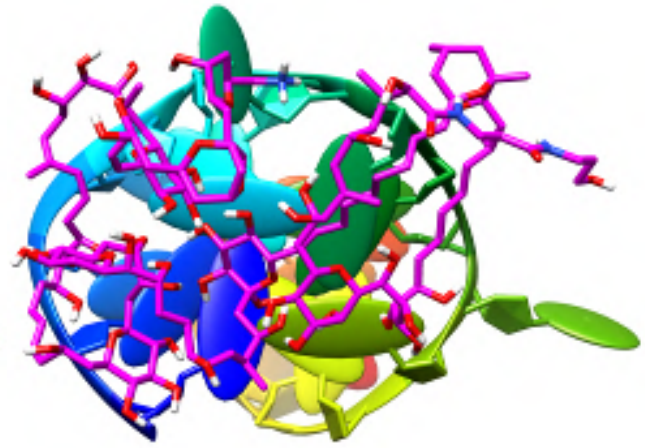

C
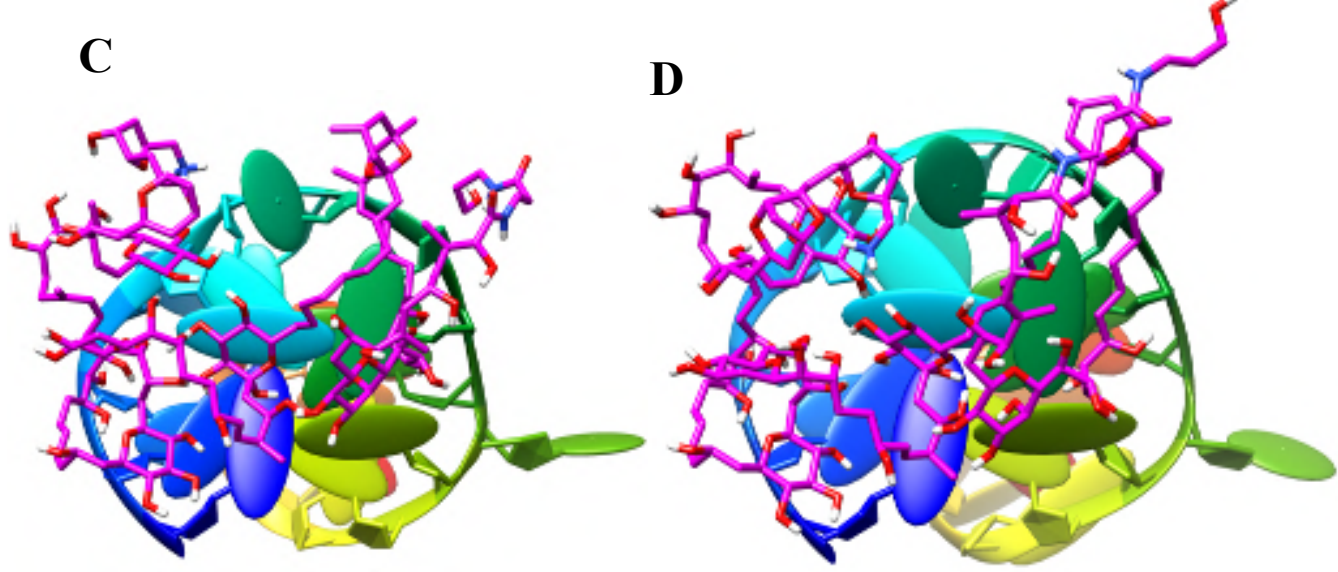

Figure 4 Top-view of the conformations of complex P-18S2-PTX versus MD simulation time

A 0ns, B 10ns, C 20ns, D 30ns 
A

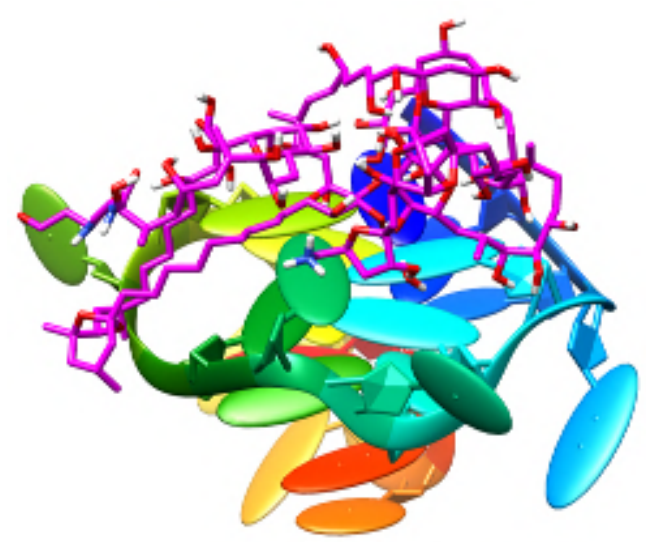

C

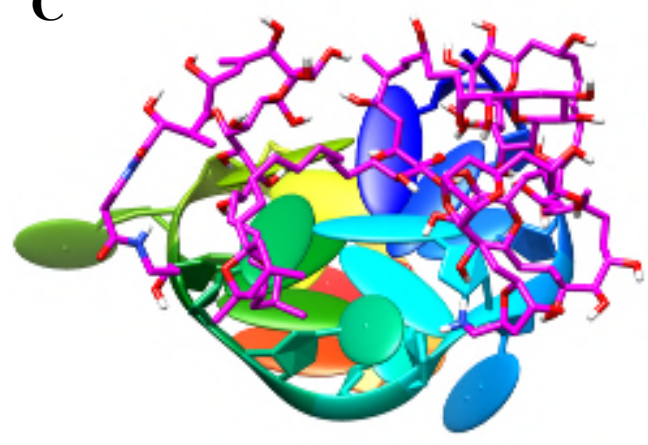

B

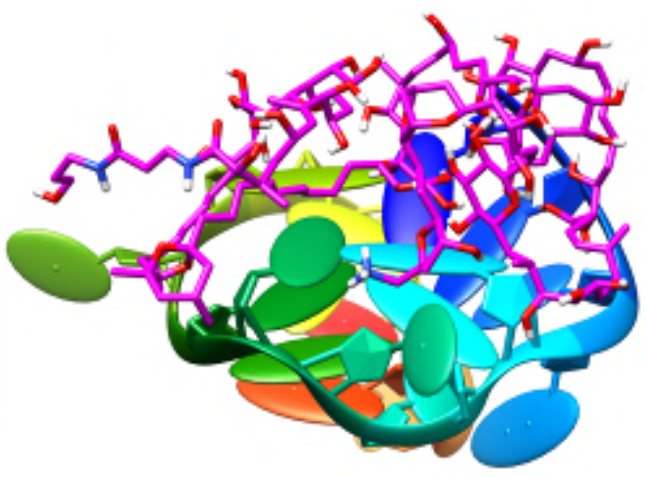

D

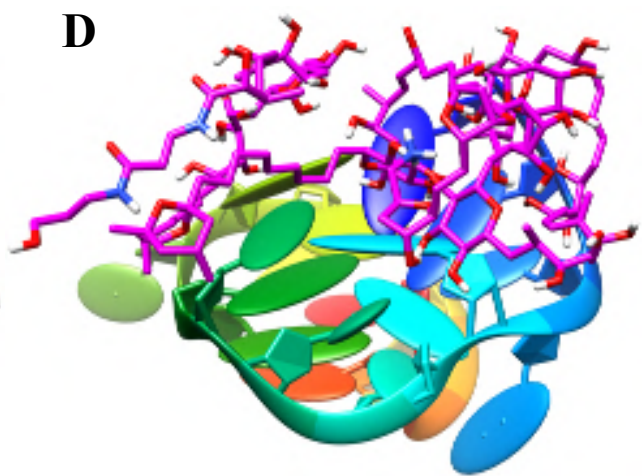

Figure 5 Oblique-view of the conformations of complex P-18S2-PTX versus MD simulation time

A 0ns, B 10ns, C 20ns, D 30ns

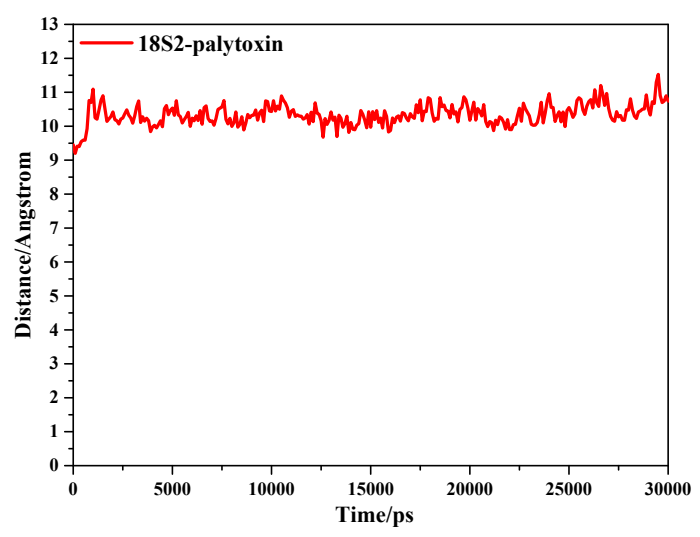

Figure 6 Mass distance between P-18S2 and PTX versus simulation time 


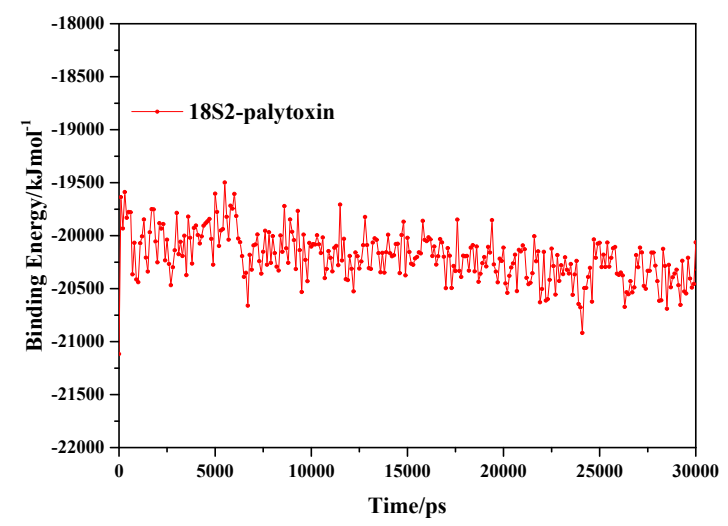

Figure 7 Binding energy of complexes versus simulation time

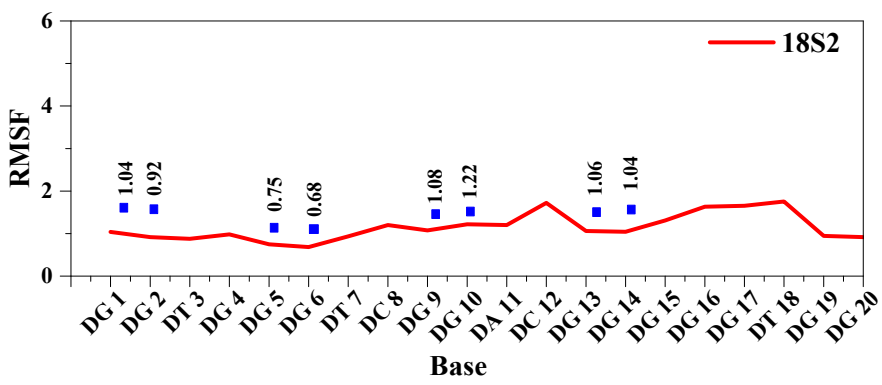

Figure 8 RMSF of residues of complex versus simulation time

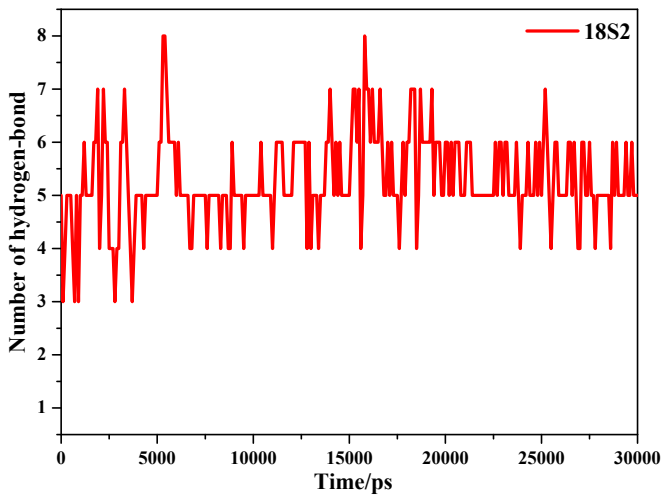

Figure 9 Hydrogen bonds between P-13S2, P-18S2 and PTX versus simulation time
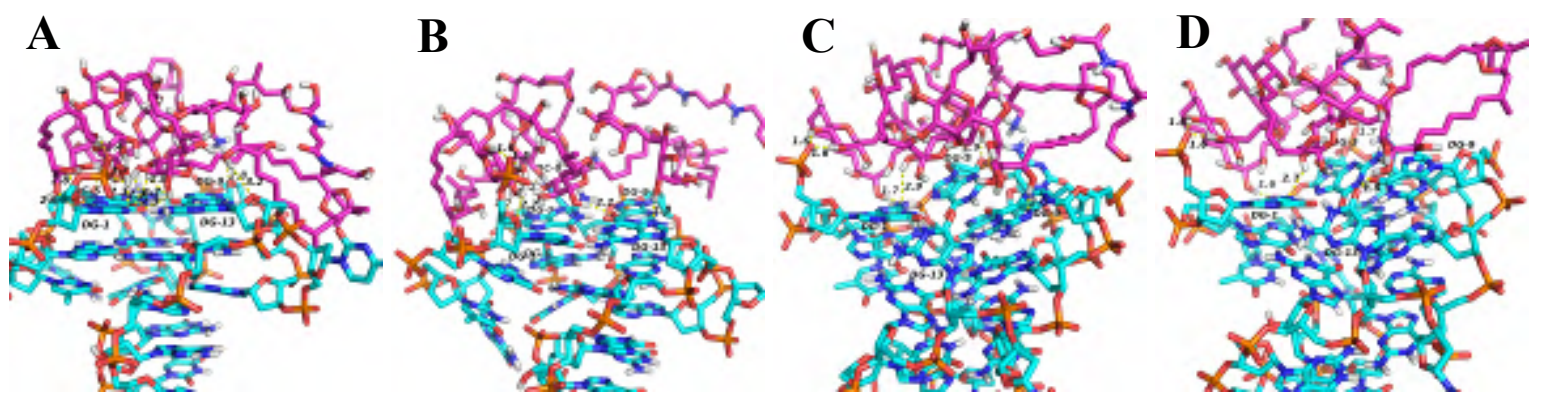

Figure 10 3D-view of hydrogen bonds interaction between P-18S2 and PTX versus simulation time 


\section{A 0ns, B 10ns, C 20ns, D 30ns}

The stability of G-quadruplex structures were analyzed as well, shown in S1 Fig. As we can see, the G-quadruplex structures of DNA P-18S2 show little fluctuation in the simulation, indicated that DNA P-18S2 with G-quadruplex structure has a strong intramolecular interaction, lead to the stability of G-quadruplex structure. As a result, the root mean square fluctuation (RMSF) (Fig 8) of the key bases of G-quadruplex structure, such as G1, G2, G5, G6, G9, G10, G13 and G14 in P$18 S 2$, showed a small value less than $2 \AA$. So it can be concluded that DNA with G-quadruplex structure keep steady in the progress of MD simulation, led to the stable combination between PTX and DNA. After quantitative analysis, it is found that the RMSF of the key bases of G-quadruplex structure $(\mathrm{G} 1, \mathrm{G} 2, \mathrm{G} 5, \mathrm{G} 6, \mathrm{G} 9, \mathrm{G} 10, \mathrm{G} 13$ and $\mathrm{G} 14$ with a value of $1.03,0.91,0.74,0.68,1.07,1.21$, 1.05 and $1.04 \AA$ respectively) from P-18S2 is very low. That is why P-18S2 shows high activity for blocking the binding of PTX to Sodium channel protein.

In order to give a new insight into the binding of DNA with PTX during the MD simulation, the hydrogen bonds were monitored, shown in Fig 9. The plots of the hydrogen bonds between DNA and PTX indicated that the DNA combined with PTX stably by the strong hydrogen bonds interaction. In the process of dynamic simulation, the hydrogen bonds of the complexes always exist. Some hydrogen bonds exist only for a period of time, named dynamic hydrogen bonds, while others, called static hydrogen bond, persisted through the MD simulations. The analysis of the dynamic behavior of hydrogen bonds was performed to explore the blocking mechanism of DNA for the combination of PTX and sodium channel protein. The three-dimensional structures of the interaction of DNA P-18S2 with PTX system was visualized in Fig 10. It is found that there are both static hydrogen bonds and dynamic hydrogen bonds between DNA P-18S2 and PTX during the dynamic simulation. As shown in Fig 10, there are three static hydrogen bonds and eighteen dynamic hydrogen bonds generated between P-18S2 and PTX, made the binding of P-18S2 and PTX more stable and more active to block of the binding of PTX to Sodium channel protein.

Strong hydrogen bonds interaction promoted the stability of the complexes. The trajectory diagram of radius of gyration also proved this point. The radius of gyration of biomacromolecular is a sign of stability in the process of dynamic simulation. As we can be seen from trajectory diagram of radius of gyration from the complexes P-18S2-PTX (Fig 11), radius of gyration is stable after $5 \mathrm{~ns}$ and the fluctuation value is negligible. Therefore, the radius of gyration of complex further validated the stable DNA with G-quadruplex structure and combination between DNA and PTX. 


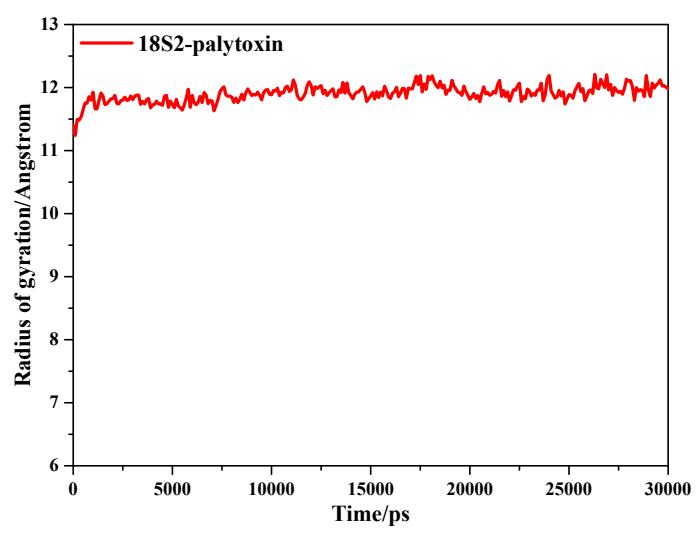

Figure 11 Radius of gyration of complex versus simulation time

\section{Affinity of between truncation of P-18S2 and PTX}

The BLI results showed that PTX interacted with truncated successively truncated sequence P18-S3,P18-S4, P18-S5, P18-S6, P18-S7, P18-S8 with a Kd (nM) of 3.14, 2.13, 0.87, 0.81, 2.93, 2.62 respectively, and P18-S2 with a Kd of $1.09 \mathrm{nM}$, shown in S2 Table and S2 Fig. Those data very close to the previous result $(0.93 \mathrm{nM})^{[10]}$, and no phenomenon were observed in the corresponding blank or negative controls, which demonstrated that there is no significant difference between truncated P-18S2 and primary P-18S2 to bind PTX. In addition, this result further verified the reliability of computer simulation and defined the most core site of P-18S2 and PTX.

\section{Methods}

\section{G-quadruplex model generation}

The structure prediction of P-18S2 was performed with QGRS(quadruplex forming G-rich sequences, $h$ ttp://bioinformatics.ramapo.edu/QGRS/index.php).Minimum G-Group size was settled as 2. There are 56 possible structures for P-18S2 in forming G-quadruplex structure, the top 10 results of QGRS prediction were showed in S3 Table, with a maximum G-Score of 21. In a word, P$18 \mathrm{~S} 2$ was great possibility in formation of G-quadruplex structure.

Then, the search in the Nucleic Acid Database (NDB) ${ }^{[20]}$ for quadruplex DNA structures returned 193 entries. Unfortunately this list did not include all G-quadruplex containing structures. The Protein Data Bank (PDB) ${ }^{[21]}$ was searched as well for entries containing the words "quadruplex" and the list was filtered by visual inspection. The sequences extracted for each chain in the corresponding $\mathrm{PDB}$ files were aligned to $\mathrm{P}-18 \mathrm{~S} 2$. The $2 \mathrm{LXQ}{ }^{[22]}$ was found that it was exhibiting a corresponding intrastrand G-quadruplexes and similar size to P-18S2. In addition, the arrangement of guanine in $2 \mathrm{LXQ}$ was similar to $\mathrm{P}-18 \mathrm{~S} 2$ to a certain extent. Based on the atomic models of $2 \mathrm{LXQ}$, the $3 \mathrm{D}$ model of $\mathrm{P}-18 \mathrm{~S} 2$ with G-quadruplex structure was generated by Discovery Studio 2.5 Client (http://accelrys.com/products/discovery-studio) with the nucleic acids substitution, insertion and deletion.

Care was taken to make the coordination geometry most favorable. Conformations of a few nucleic acids were therefore adjusted manually within well allowed ranges. Then, the optimization of model was performed at the high performance computing facility with the YASARA package ${ }^{[23,24]}$, using the Amber 14 force field ${ }^{[2]}$, and the water model was TIP3P. The temperature coupling of the model system was ascertained by Berendsen thermostat method, while the manometer method 
was used for pressure coupling ${ }^{[26]}$. Besides, the starting structure was immersed in a periodic rectangular simulation cubic cell of water. For the operation of optimization in the simulated water condition, the backbone was firstly fixed and the side chain was optimized 5,000 steps, and then, the whole structure was majorized 5,000 steps. Finally, the optimized 3D-struture of P-18S2 with Gquadruplex structure was generated, shown in Fig 12. After optimization, quantitative analysis was performed to the G-quadruplex structure of P-18S2. The results showed that, due to strong hydrogen-bonds interaction among G1, G5, G9, G13 and G2, G6, G10, G14 in P-18S2 (S3 Fig), formed stable G-quadruplex structure.
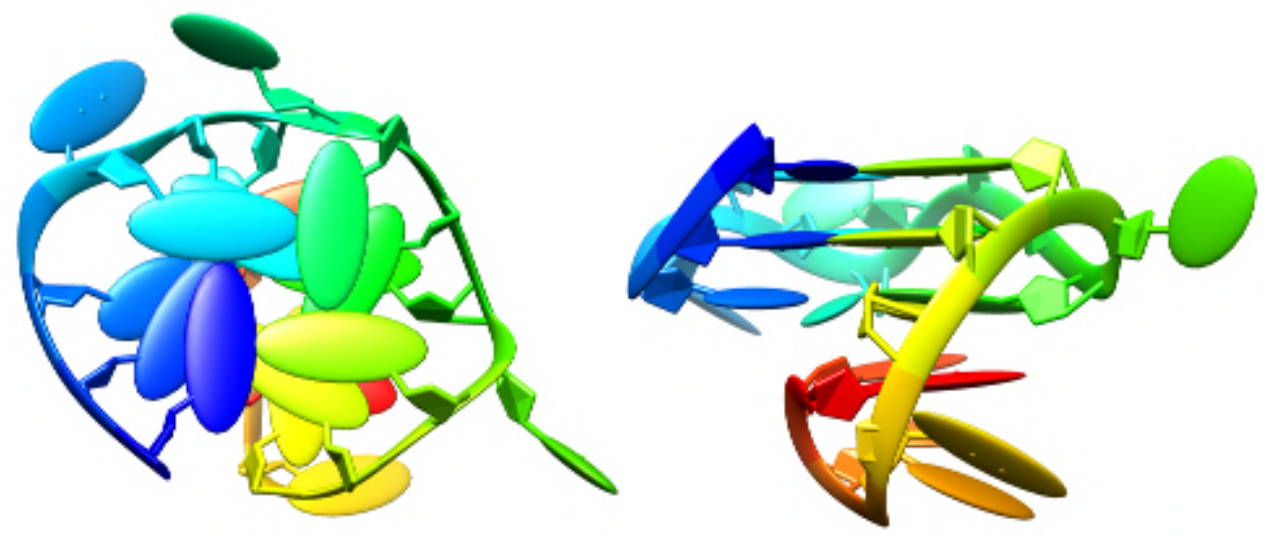

Figure 12 The 3D model of P-18S2 with G-quadruplex structure, top view at left and side view at right

\section{Docking}

Firstly, PTX was obtained from PubChem Compound database with accession no. 45027797, shown in S4 Fig. The PTX molecule was optimized using MM2 method. Due to large size and high molecular weight, PTX showed high flexibility and lots of possible conformations. So, it is unreasonable to perform the docking using PTX from the database directly. The solution to these problems is to first obtain the equilibrium structure of PTX in the solvent and then use it for molecular docking. Only in this way can we be able to investigate the interaction between PTX and DNA P-18S2 scientifically. In this work, 30 ns dynamics simulation is performed to PTX in $\mathrm{KCl}$ aqueous solution using GAFF force field[27], then repeat three times. It was found that in the process of dynamic simulation the PTX tended to constriction in aqueous solution and reach an equilibrium state, shown in Fig 13. The 3D structures of PTX after equilibrium were output and optimized. Subsequently, the total energy was calculated, and the structure with lowest energy was selected to use for the subsequent simulations. Then, the number of rotatable bonds of ligands was set up in AutodockTools ${ }^{[28]}$. Finally, PTX was output and saved as the ligand. 
A

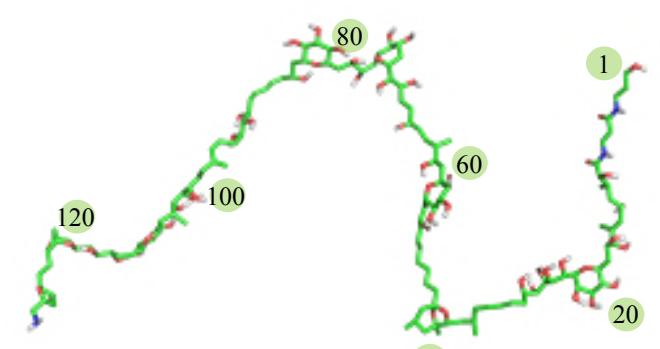

40
B

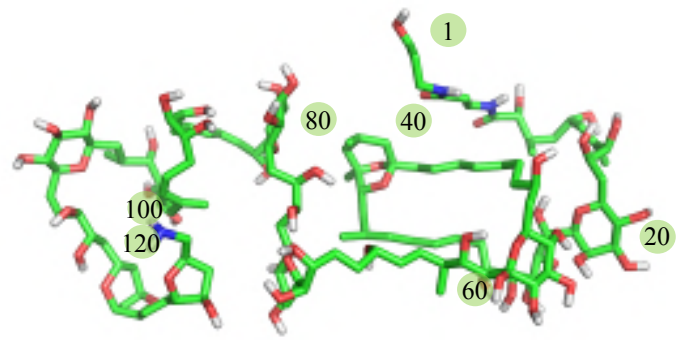

C

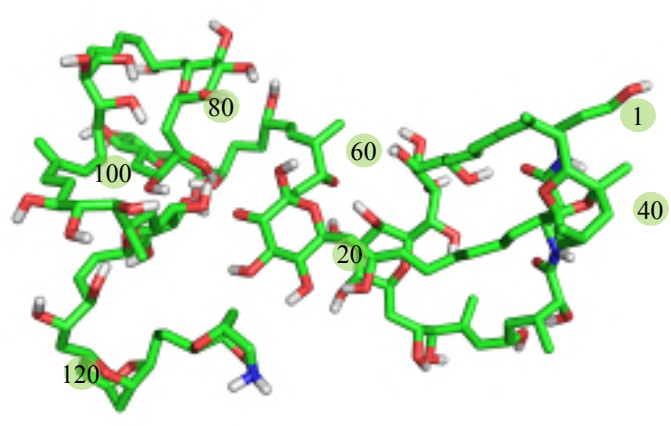

D

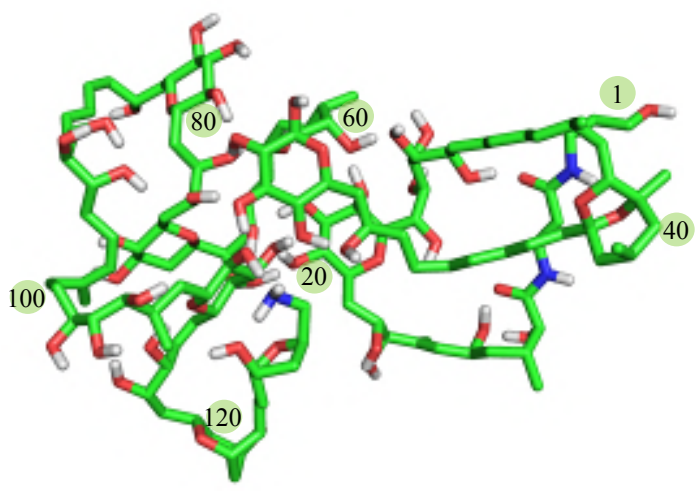

Figure 13 Conformation change of the PTX in the process of dynamic simulation :(A) 0ns, (B) 10ns, (C) 20ns, (D) 30ns

For DNA-ligands docking, 3D structure of P-18S2 was obtained from the modelling and initialized as receptor molecules with AutodockTools. Subsequently, the receptor was endowed with $A D$ atomic type, and hydrogen and charge were added, followed by the mergence of nonpolar hydrogen. The binding sites of P-18S2 recognizing ligand was obtained based on crystal structures of DNA-ligands complex with G-quadruplex structure ${ }^{[29-33]}$, which proved that the binding site of DNA with G-quadruplex structure were in the groove at the top and the bottom. In addition, the experiment suggested that the ligand could induce the formation of G-quadruplex structure. Therefore, as demonstrated in our previous work ${ }^{[17]}$, the binding site of P-18S2 with G-quadruplex structure was at the top (S5 Fig).

Finally, the molecular docking analysis was carried out using Hex 8.0[34]. Rotation of receptor and ligand was allowed in 180 degree, without the specified binding group. The shape and electrostatic interaction were also considered. The maximum number of docking was revised as 200 and cluster analysis were made, while the default parameter settings were retained for others. For each of the docking cases, the lowest energy conformation, according to the Hex scoring function, 
was selected as the binding mode. The output from Hex was rendered with Chimera ${ }^{[35]}$ and PyMol program ${ }^{[36]}$.

\section{Molecular dynamics simulations}

The Molecular dynamics (MD) simulations carried out were based on the complex which obtained from docking in Hex program. All simulations were performed using the molecular dynamics program YASARA and the amber 14 force field ${ }^{[37]}$. The complex using in the simulation came from molecular docking with hydrogen generated by the YASARA program. All simulations were carried out with an integration step of $1 \mathrm{fs}$ and coordinates of the simulation model were recorded per $1 \mathrm{ps}$. The starting structures were immersed in a periodic rectangular simulation cubic cell of $\mathrm{KCl}$ aqueous solution. The box dimensions were chosen to provide at least a $10 \AA$ buffers of solvent molecules around the solute.

The fully solvated systems were then subjected to 5000 steps steepest descent minimization runs to remove clashes between atoms. An 80 ps position restrained MD simulation was performed for each system at constant pressure $(1 \mathrm{~atm})$ and temperature $(300 \mathrm{~K})$. The temperature and pressure were kept constant during the simulations. Temperature coupling was done using the Berendsen thermostat with a temperature coupling constant of $0.1 \mathrm{ps}$, while the manometer method was used for pressure coupling, with a reference pressure of $1 \mathrm{~atm}$. A particle mesh Ewald scheme $^{[38,39]}$ was used to calculate the long range electrostatic interactions with a $10 \AA$ cutoff for the real space. A cutoff of $14 \AA$ was used for the van der Waals interactions (Lennard-Jones terms).Translation and rotation corrections were enabled during MD simulations to ensure that structures in trajectory were well superimposed, which is convenient for the structure analysis. The chemical bond lengths involving hydrogen atoms were fixed with SHAKE algorithm ${ }^{[40]}$.

By the time of $1 \mathrm{~ns}$, the simulation system reached an equilibrium state; thus, the system was subjected to conventional MD (CMD) simulation for $30 \mathrm{~ns}$. All calculations were performed on the MolDesigner molecular simulation platform.

\section{Determination of affinity by BLI}

We surprised to found that P18-S2 can be further truncated and optimized by simulating and docking. Therefore, the affinity of the successively truncated sequence (P18-S3 P18-S8) and P18S2 for binding to PTX were determined respectively. The super streptavidin-coated (SSA) biosensor was used for the immobilization of the 7 sequences onto the BLI aptasensor. Those aptamers were analyzed for association time over 2 min with PTX(5 uM) and dissociation time over 2 min, along with a blank sample containing only binding buffer for reference and a random sequence was used as a negative control.

\section{Acknowledgements}

We are sincerely grateful for Chengdu FenDi Technology on technical guidance in computer simulation.

\section{References}

1.Moore RE, Scheuer PJ. Palytoxin: a new marine toxin from a coelenterate. Science. 1971;172(3982):495-8. 
2.Volpe G, Cozzi L, Migliorelli D, Croci L, Palleschi G. Development of a haemolytic-enzymatic assay with mediated amperometric detection for palytoxin analysis: application to mussels. Analytical \& Bioanalytical Chemistry. 2014;406(9-10):2399-410.

3.Louzao MC, Ares IR, Cagide E. Marine toxins and the cytoskeleton: a new view of palytoxin toxicity. Febs J. 2008;275(24):6067-74. doi: 10.1111/j.1742-4658.2008.06712.x. PubMed PMID: WOS:000261184700003.

4.Deeds JR, Schwartz MD. Human risk associated with palytoxin exposure. Toxicon : official journal of the International Society on Toxinology. 2010;56(2):150-62. doi: 10.1016/j.toxicon.2009.05.035. PubMed PMID: WOS:000279065100005.

5.Tubaro A, Durando P, Del Favero G, Ansaldi F, Icardi G, Deeds JR, et al. Case definitions for human poisonings postulated to palytoxins exposure. Toxicon : official journal of the International Society on Toxinology. 2011;57(3):478-95. doi: 10.1016/j.toxicon.2011.01.005. PubMed PMID: 21255599.

6.Riobó P, Paz B, Franco JM, Vázquez JA, Murado MA, Cacho E. Mouse bioassay for palytoxin. Specific symptoms and dose-response against dose-death time relationships. Food \& Chemical Toxicology. 2008;46(8):2639-47.

7.Ciminiello P, Dell'Aversano C, lacovo ED, Fattorusso E, Forino M, Tartaglione L. LC-MS of palytoxin and its analogues: State of the art and future perspectives. Toxicon : official journal of the International Society on Toxinology. 2011;57(3):376-89.

8.Sophie KA, Zouher A, Ralph P, Stjepko G, Manoella S, Taiana DH, et al. First Evidence of Palytoxin and 42-Hydroxy-palytoxin in the Marine CyanobacteriumTrichodesmium. Marine drugs. 2011;9(4):543-60.

9.Riob, Oacute P, Paz B, Franco JM. Analysis of palytoxin-like in Ostreopsis cultures by liquid chromatography with precolumn derivatization and fluorescence detection. Analytica Chimica Acta. 2006;566(2):217-23.

10.Gao S, Zheng X, Hu B, Sun M, Wu J, Jiao B, et al. Enzyme-linked, aptamer-based, competitive biolayer interferometry biosensor for palytoxin. Biosensors \& bioelectronics. 2017;89(Pt 2):952-8.

11.Ellington AD, Szostak JW. In vitro selection of RNA molecules that bind specific ligands. Nature. 1990;346(6287):818-22.

12.Patel DJ, Jiang F, Jiang LFP, Kumar RA, Nonin S, Suri AK. STRUCTURE, RECOGNITION AND ADAPTIVE BINDING IN RNA APTAMER COMPLEXES [Review]. Journal of molecular biology. 1997;272(5):645-64.

13.Graziella C, Franca C. Dynamic Nature of Noncoding RNA Regulation of Adaptive Immune Response. International journal of molecular sciences. 2013;14(9):17347-77.

14. Handy SM, Yakes BJ, Degrasse JA, Campbell K, Elliott CT, Kanyuck KM, et al. First report of the use of a saxitoxin-protein conjugate to develop a DNA aptamer to a small molecule toxin. Toxicon Official Journal of the International Society on Toxinology. 2013;61(1):30-7.

15.Gao S, Hu B, Zheng X, Cao Y, Liu D, Sun M, et al. Gonyautoxin 1/4 aptamers with high-affinity and high-specificity: From efficient selection to aptasensor application. Biosensors \& bioelectronics. 2016;79:938-44.

16.Ouyang S, Hu B, Zhou R, Liu D, Peng D, Li Z, et al. Rapid and sensitive detection of nodularin-R in water by a label-free BLI aptasensor. Analyst. 2018;143(18):4316-22. doi: 10.1039/c8an00567b. PubMed PMID: 30101954.

17.Gao SX, Hu B, Zheng X, Liu DJ, Sun MJ, Qin JX, et al. Study of the binding mechanism between aptamer GO18-T-d and gonyautoxin $1 / 4$ by molecular simulation. Physical Chemistry Chemical Physics. 2016;18(34):23458-61. doi: 10.1039/c6cp00777e. PubMed PMID: WOS:000382107200002. 18.Concepcion J, Witte K, Wartchow C, Choo S, Yao DF, Persson H, et al. Label-Free Detection of Biomolecular Interactions Using BioLayer Interferometry for Kinetic Characterization. Combinatorial Chemistry \& High Throughput Screening. 2009;12(8):791-800. doi: Doi 10.2174/138620709789104915. PubMed PMID: WOS:000271343000006.

19.Ritchie DW, Kemp GJL. Protein docking using spherical polar Fourier correlations. Proteinsstructure Function \& Bioinformatics. 2015;39(2):178-94. 
20.Berman HM, Olson WK, Beveridge DL, Westbrook J, Gelbin A, Demeny T, et al. The nucleic acid database. A comprehensive relational database of three-dimensional structures of nucleic acids. Biophysical Journal. 1992;63(3):751-9.

21.Berman HM, Westbrook J, Feng Z, Gilliland G, Bhat TN, Weissig H, et al. The Protein Data Bank/ Nucleic Acids Research. Nucleic Acids Research. 2000;17(20):3588.

22.Kuryavyi V, Cahoon LA, Seifert HS, Patel DJ. RecA-binding pilE G4 sequence essential for pilin antigenic variation forms monomeric and $5^{\prime}$ end-stacked dimeric parallel G-quadruplexes. Structure. 2012;20(12):2090-102.

23. Krieger E, Koraimann G, Vriend G. Increasing the precision of comparative models with YASARA NOVA\&mdash;a self - parameterizing force field. Proteins-structure Function \& Bioinformatics. 2010;47(3):393-402.

24.Krieger E, Darden T, Nabuurs SB, Finkelstein A, Vriend G. Making optimal use of empirical energy functions: force-field parameterization in crystal space. Proteins Structure Function \& Bioinformatics. 2010;57(4):678-83.

25.Cornell WD, Cieplak P, Bayly Cl, Gould IR, Merz KM, Ferguson DM, et al. A second generation force field for the simulation of proteins, nucleic acids, and organic molecules. Journal of the American Chemical Society. 1995;117(117):5179-97.

26.Berendsen HJC, Postma JPM, Gunsteren WFV, Dinola A, Haak JR. Molecular dynamics with coupling to an external bath. Journal of Chemical Physics. 1984;81(8):3684-90.

27.Wang J, Wolf RM, Caldwell JW, Kollman PA, Case DA. Development and testing of a general amber force field. Journal of Computational Chemistry. 2004;25(9):1157.

28. Huey R, Morris G M. Using AutoDock 4 with AutoDocktools: a tutorial. The Scripps Research Institute, USA, 2008: 54-56.

29.Kettani A, Bouaziz S, Wang W, Jones RA, Patel DJ. Bombyx mori single repeat telomeric DNA sequence forms a G-quadruplex capped by base triads. Nat Struct Biol. 1997;4(5):382-9.

30.Phan AT, Kuryavyi V, Gaw HY, Patel DJ. Small-molecule interaction with a five-guanine-tract Gquadruplex structure from the human MYC promoter. Nature chemical biology. 2005;1(3):167-73.

31.Parkinson GN, Ghosh R, Neidle S. Structural basis for binding of porphyrin to human telomeres. Biochemistry. 2007;46(9):2390-7.

32.Parkinson GN, Cuenca F, Neidle S. Topology conservation and loop flexibility in quadruplex-drug recognition: crystal structures of inter- and intramolecular telomeric DNA quadruplex-drug complexes. Journal of molecular biology. 2008;381(5):1145-56.

33.Bazzicalupi C, Ferraroni M, Bilia AR, Scheggi F, Gratteri P. The crystal structure of human telomeric DNA complexed with berberine: an interesting case of stacked ligand to G-tetrad ratio higher than 1:1. Nucleic Acids Research. 2013;41(1):632-8.

34.Ritchie DW, Venkatraman V. Ultra-fast FFT protein docking on graphics processors. Bioinformatics. 2010;26(19):2398-405.

35.Pettersen EF, Goddard TD, Huang CC, Couch GS, Greenblatt DM, Meng EC, et al. UCSF Chimera-a visualization system for exploratory research and analysis. J Comput Chem. 2004;25(13):1605-12. doi: 10.1002/jcc.20084. PubMed PMID: 15264254.

36.Delano WL. The PyMOL Molecular Graphics System. De-Lano Scientific, San Carlos, CA, USA. 2002.

37.Case DA, Babin V, Berryman JT, Betz RM, Cai Q,Cerutti DS, et al. Amber14; University of California:San Francisco, CA, 2014

38.Darden T, York D, Pedersen L. Particle mesh Ewald: An $N \cdot \log (\mathrm{N})$ method for Ewald sums in large systems. Journal of Chemical Physics. 1993;98(12):10089-92.

39.Essmann U, Perera L, Berkowitz ML, Darden T, Lee H, Pedersen L. A smooth particle mesh ewald method. J. Chem. Phys. 1995, 103, 8577-8593.

40.Brooks B, Bruccoleri R, Olafson B, State DJ, Swaminathan S, Karplus M. CHARMM: A program for macromolecular energy, minimization, and dynamics calculations. Journal of computational chemistry, 1983, 4(2): 187-217. 


\section{Supporting information}

S1 Fig. Oblique view of the comformations of G4 struture in DNA P-18S2 during MD simulationA 0ns, B 10ns, C 20ns, D 30ns.

S2 Fig. The red, green and blue lines respectivelyrepresent the interaction curve of aptamer18S2, 18S4, $18 S 8$ with PTX (5 uM).

S3 Fig. The G-quadruplex structure and intramolecular hydrogen bonds of P-18S2.

S4 Fig. Schematic representation of palytoxin.

S5 Fig. The binding site of P-18S2 (B) with G-quadruplex structure.

S6 Fig. The dynamic image of P-18S2 combined with PTX.

S1 Table. Docking results between DNA with G-quadruplex structure and palytoxin.

${ }^{a}$ The total calculated interaction energy; ${ }^{b}$ The original Hex steric complementarity score; ${ }^{\mathrm{c}}$ The electrostatic energy; ${ }^{\mathrm{d}}$

Root-mean-square deviation of the top-scoring docking orientation.

S2 Table. The $K_{d}$ values of aptamers combined with PTX.

S3 Table. The results of P-18S2 from QGRS prediction.

S1 File. The README file. This file includes all software and publicly available repositories along with our brief description.

S2 File. This file include input coordinates, topologies and parameter files. 
A

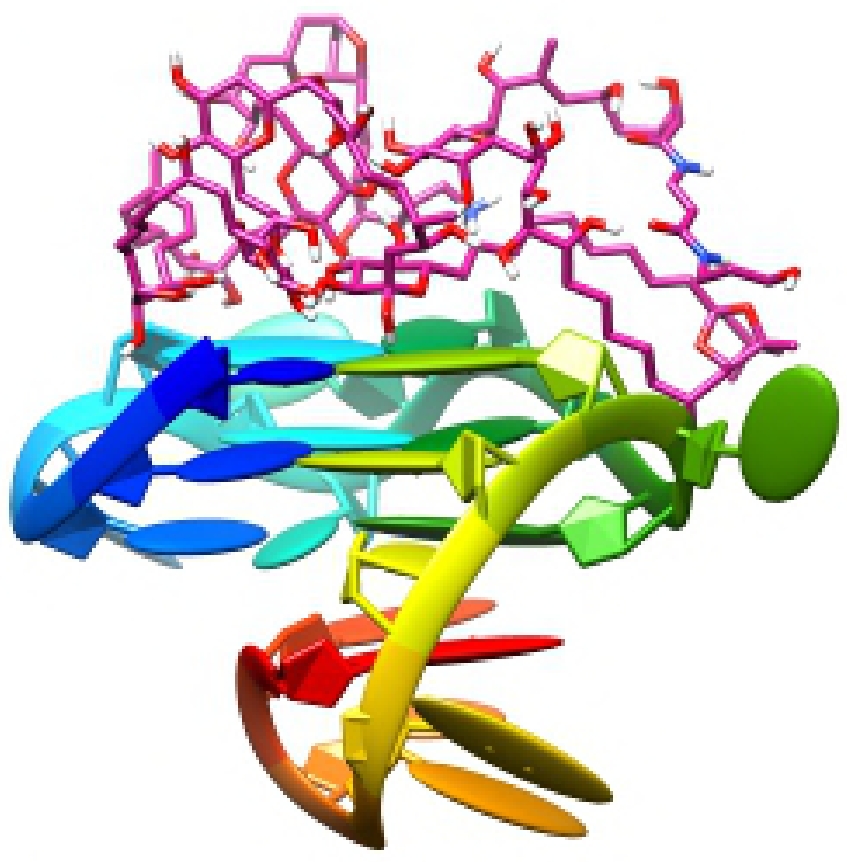

Figure 1
B

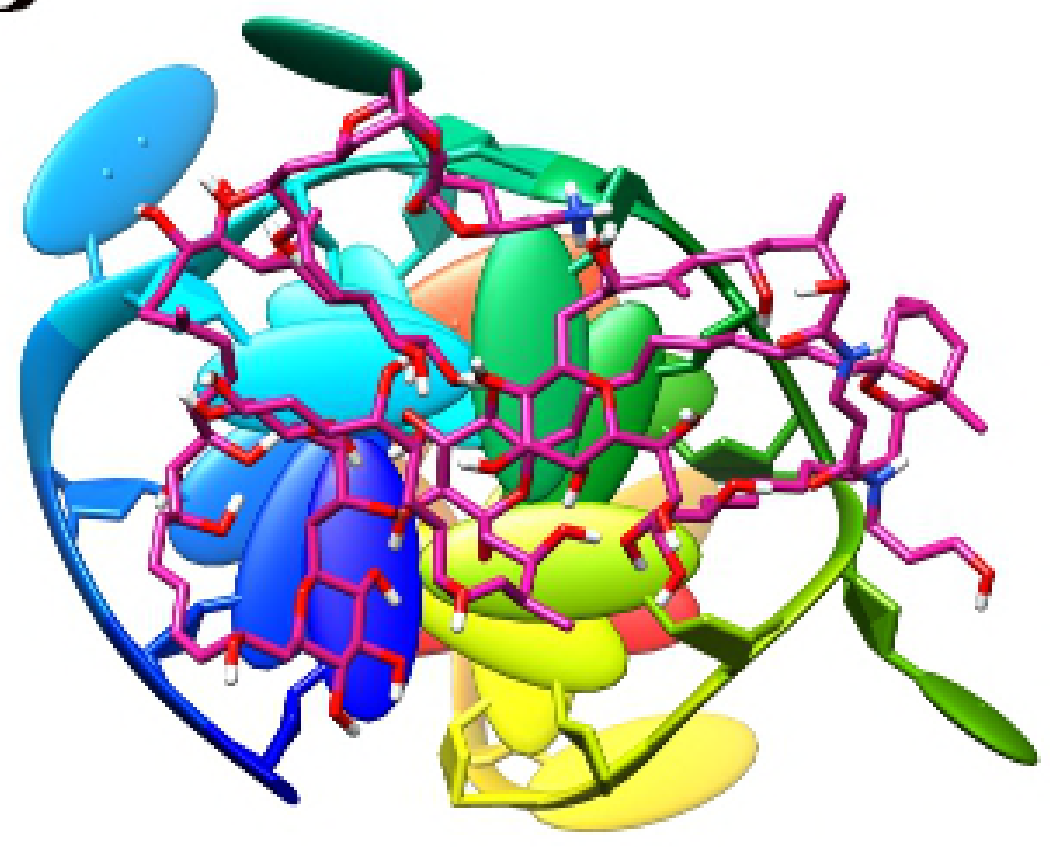



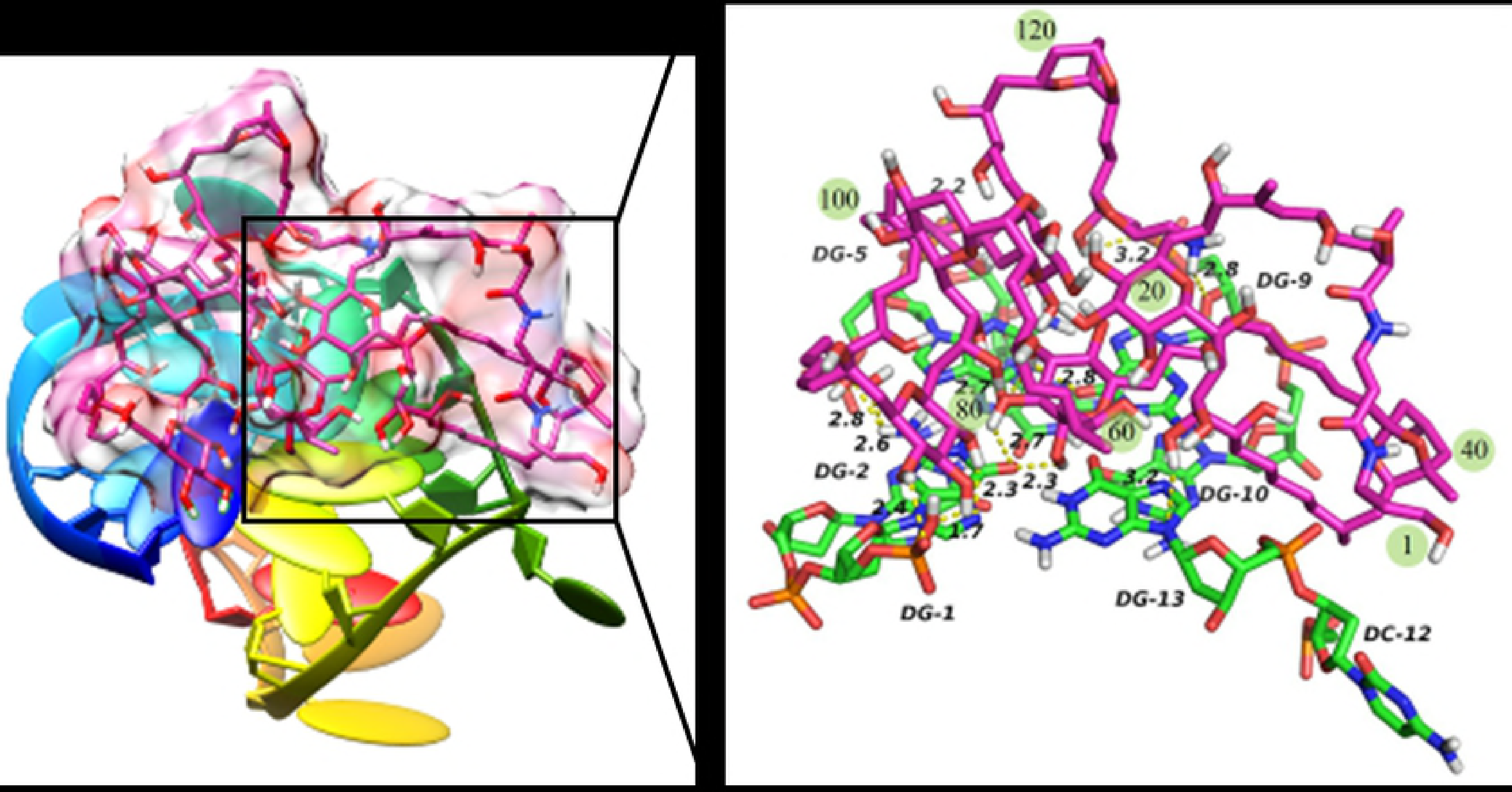

Figure 2 


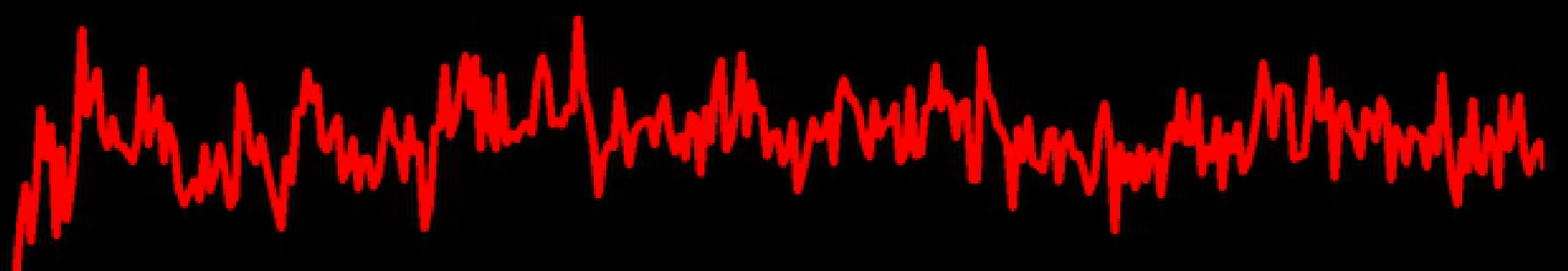

Figure 3 
A

B
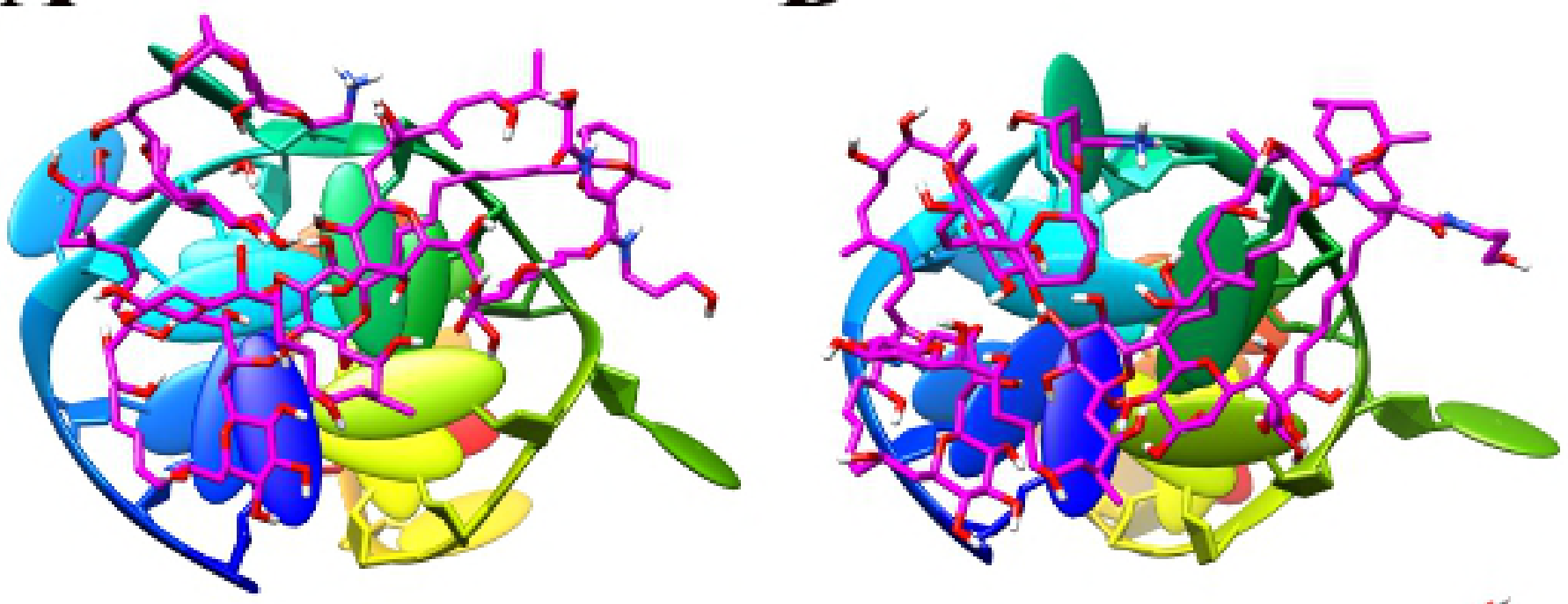

C

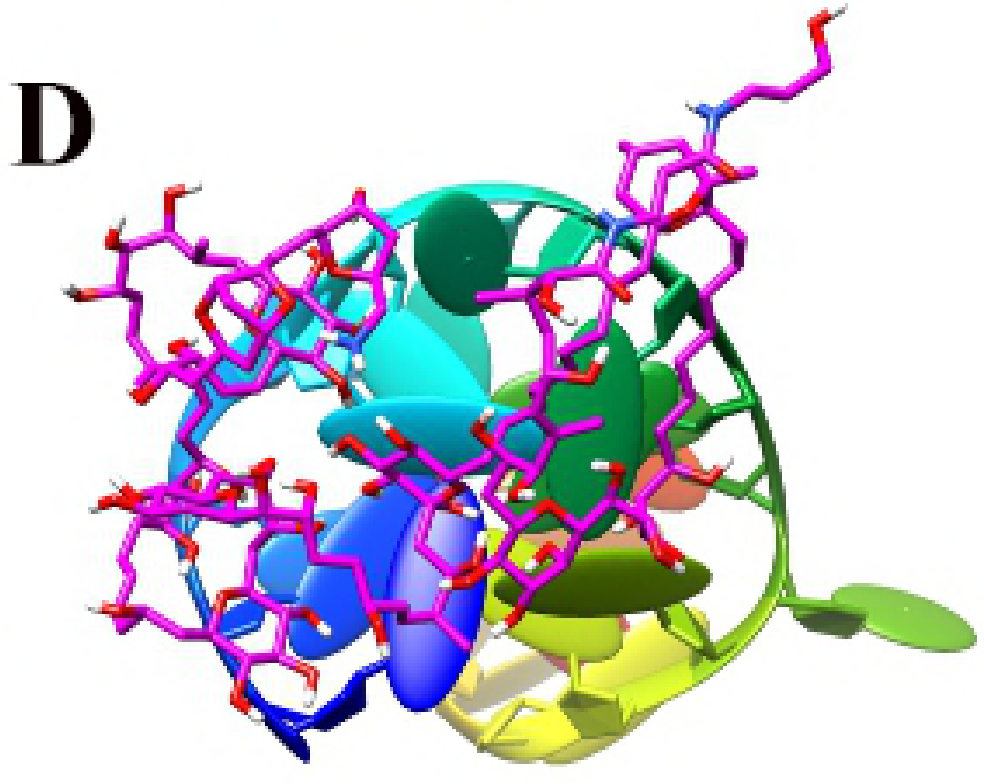

Figure 4 
A

B

mer sher 20

$5 \times 10$

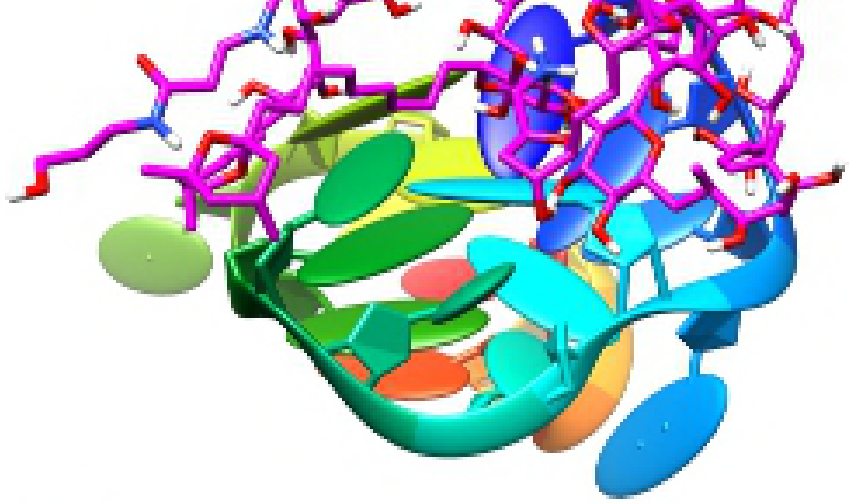

54

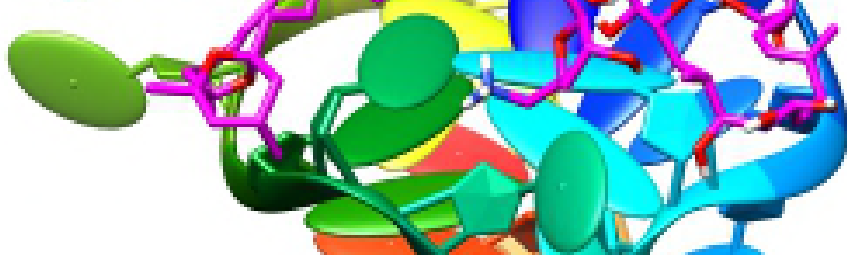

C
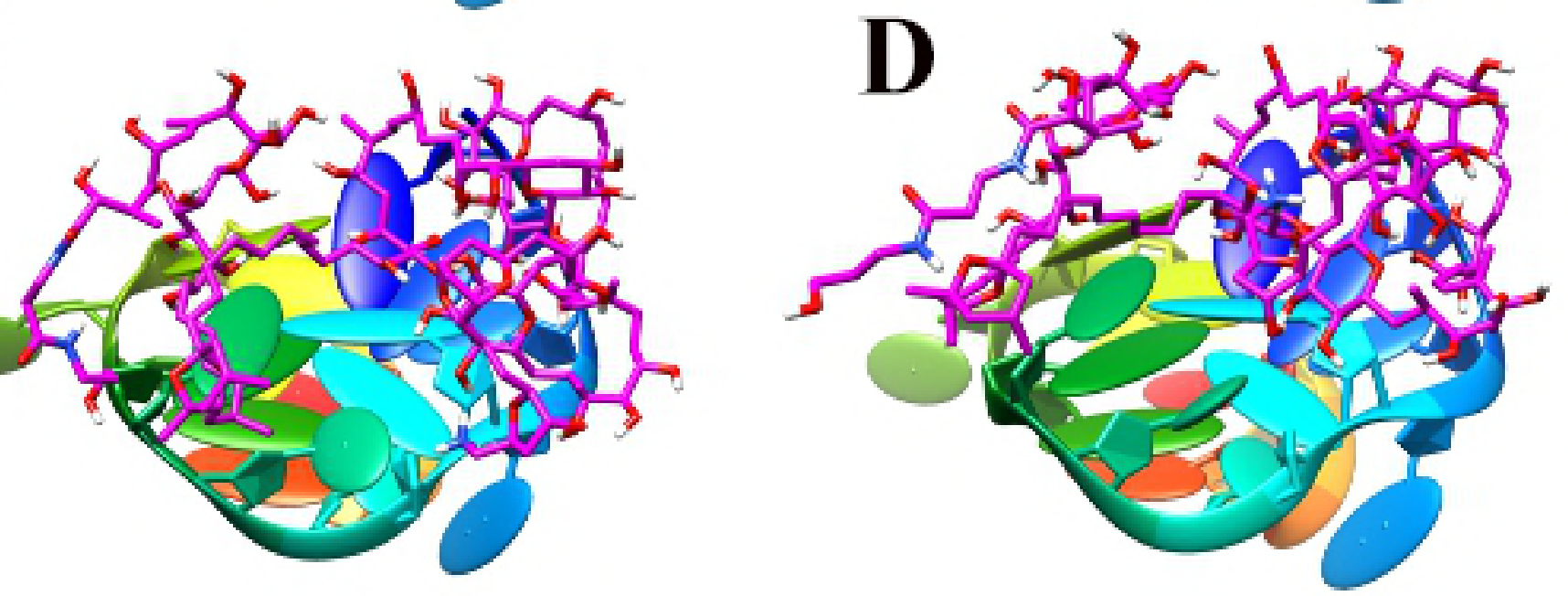

\section{Figure 5}




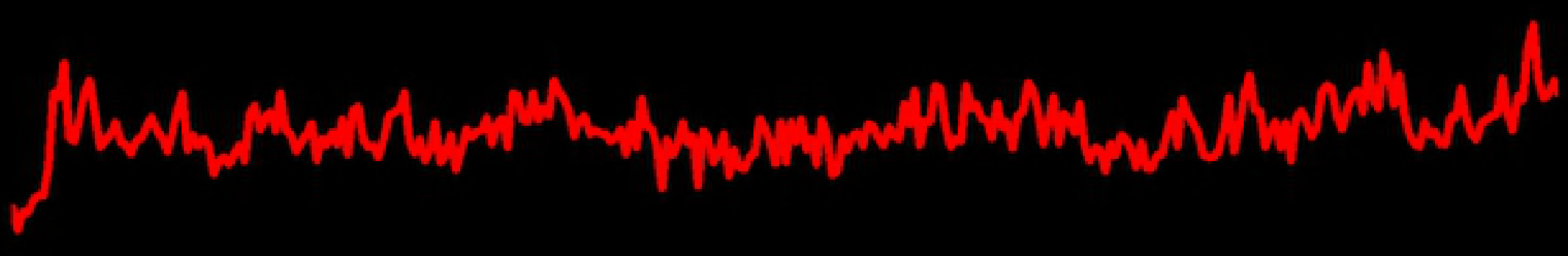

Figure 6 


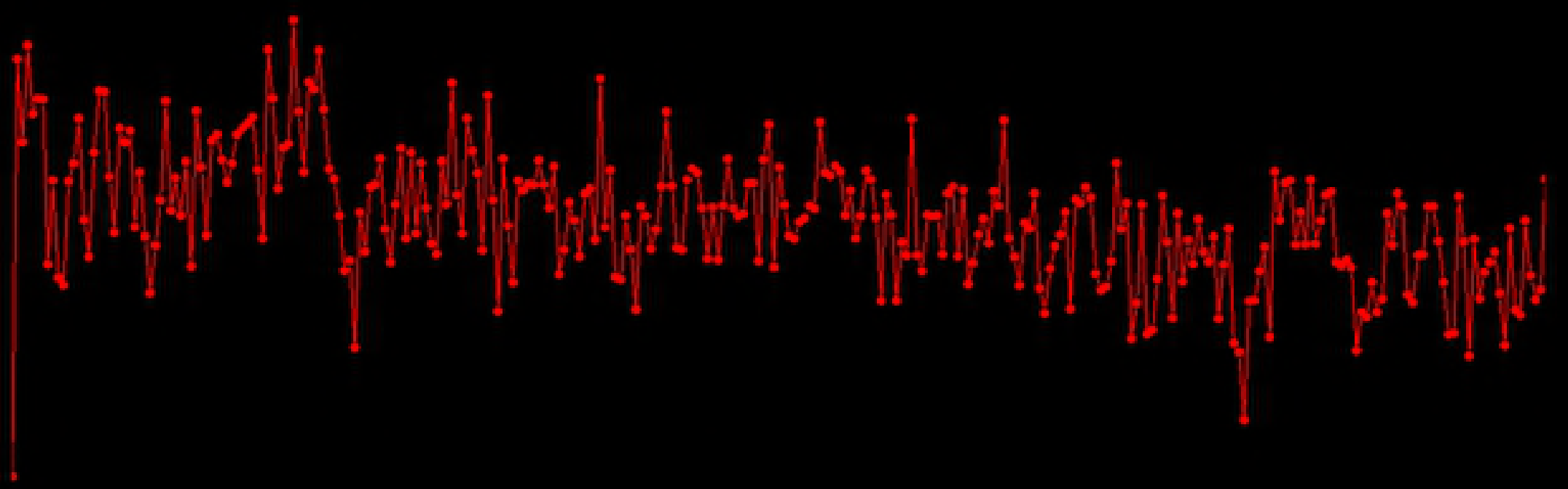

Figure 7 
Figure 8 


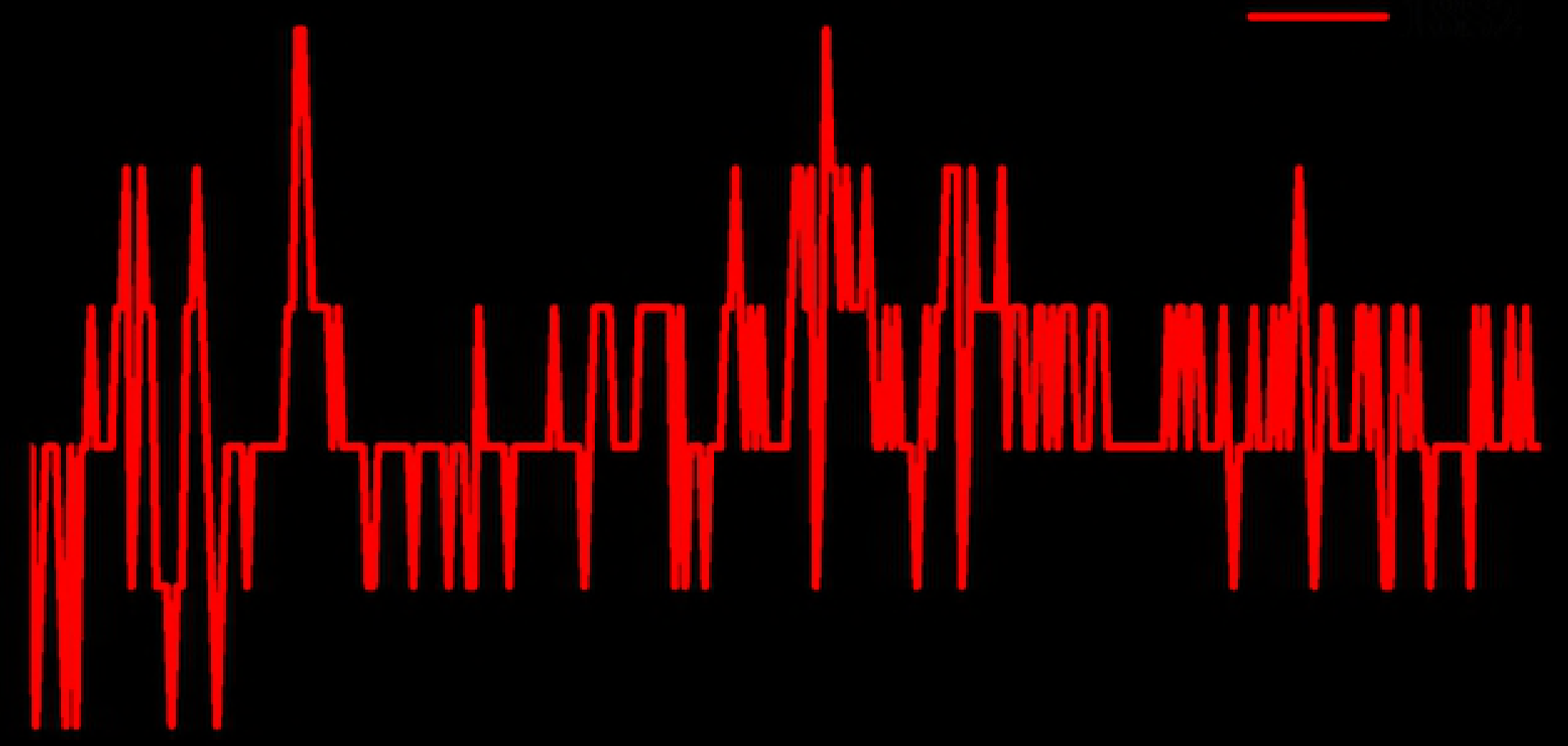

Figure 9 
A
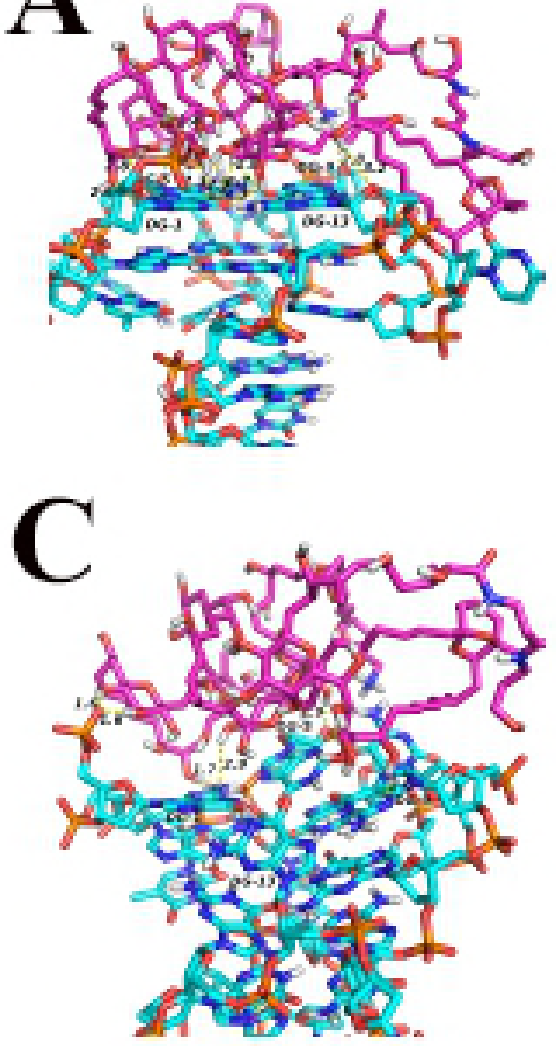

B

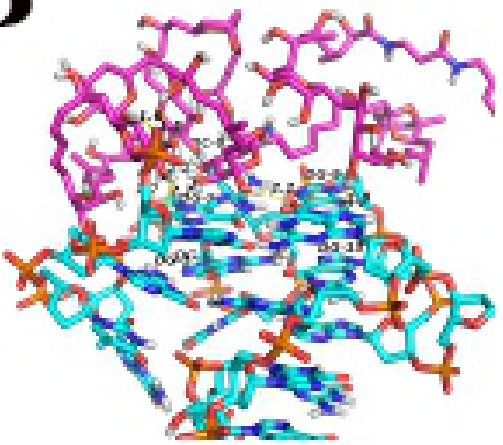

D

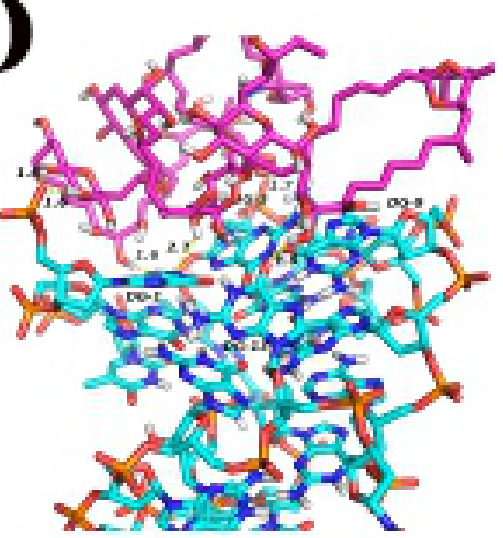

Figure 10 


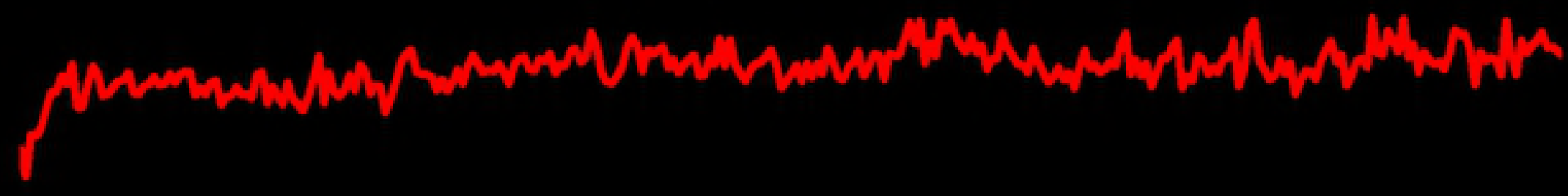

Figure 11 
$\begin{array}{ll}\text { A } & \text { B }\end{array}$
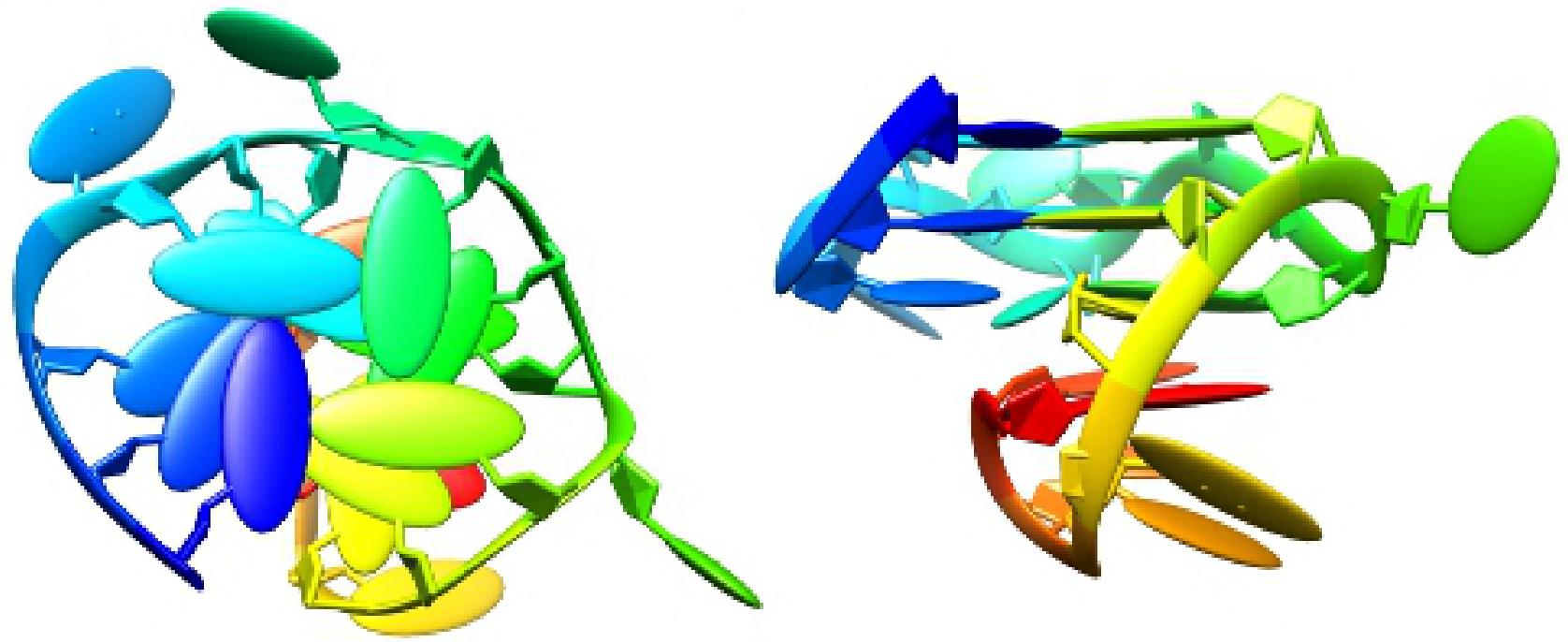

Figure 12 

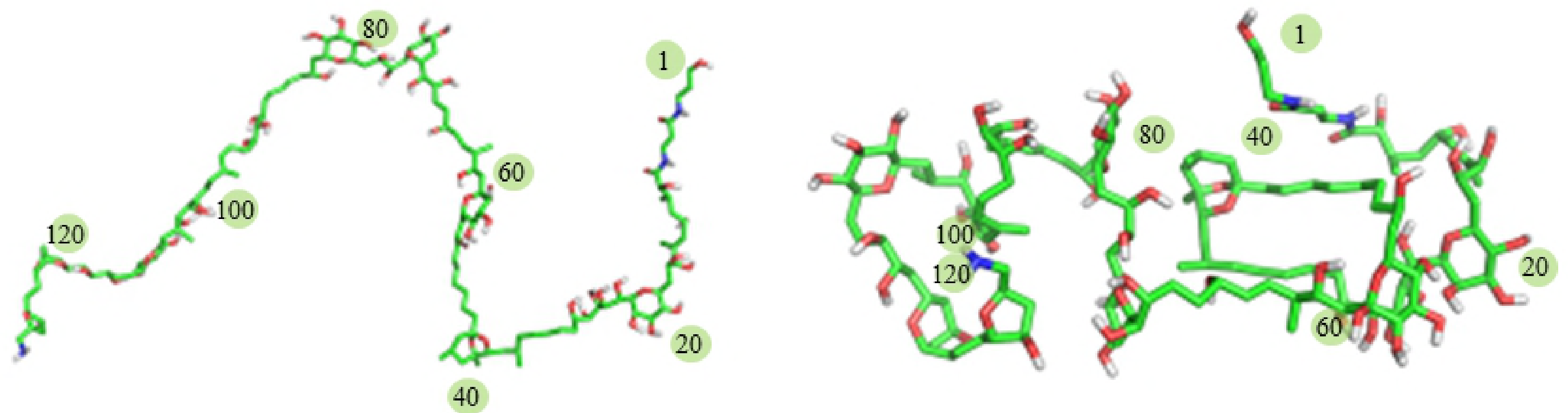

C

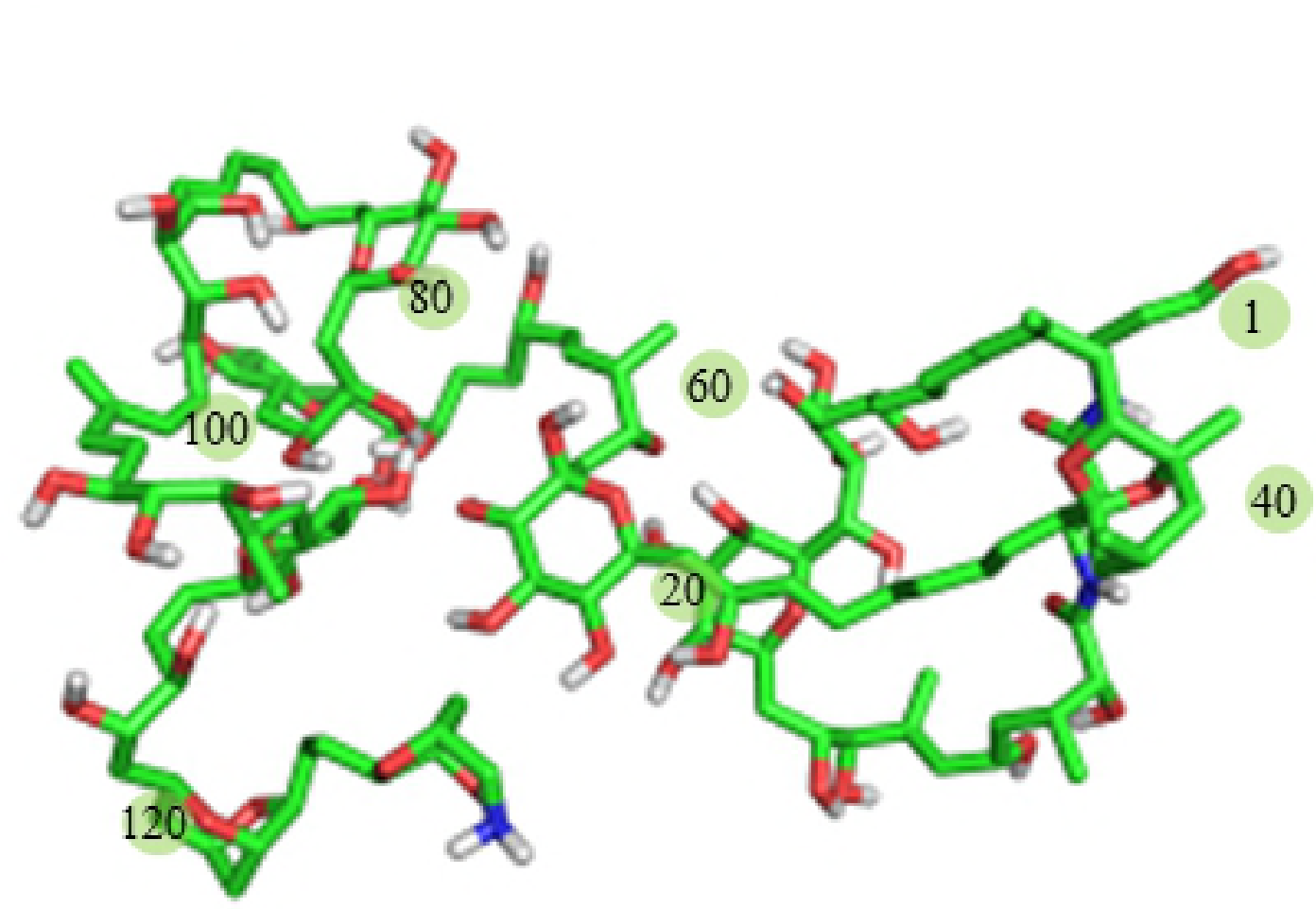

D

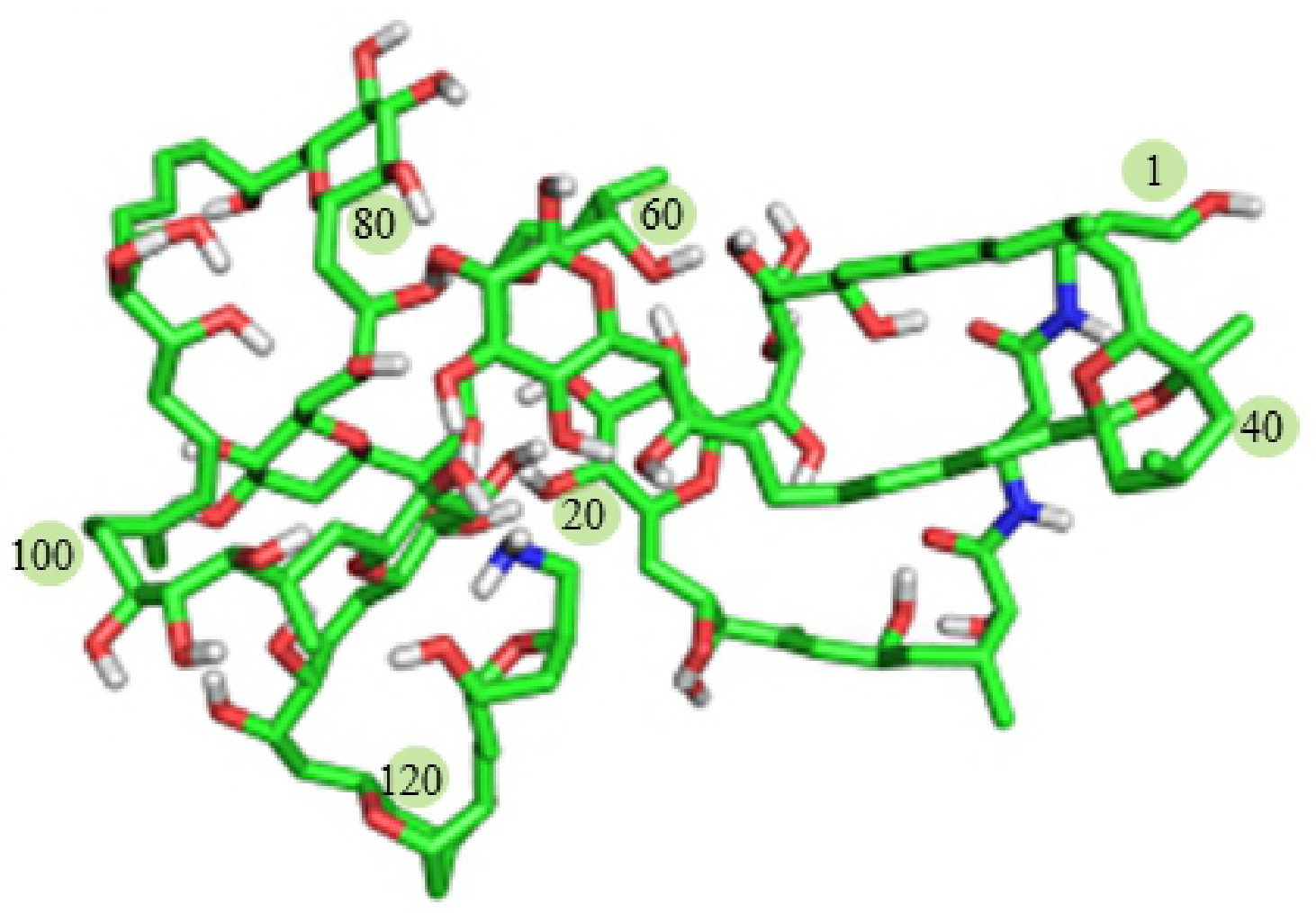

Figure 13 